\title{
ON-LINE SLURRY VISCOSITY AND CONCENTRATION MEASUREMENT AS A REAL-TIME WASTE STREAM CHARACTERIZATION TOOL
}

DE-F G 07-96E R I4727

\author{
Principal Investigator \\ Robert L. Powell \\ D epartment of Chemical $\mathbf{E}$ ngineering and $\mathrm{M}$ aterials $\mathrm{S}$ cience \\ University of California \\ Davis, CA 95616
}

\section{Co-Investigator}

Alireza Shekarriz

Pacific Northwest National Laboratories

R ichland, W A 99352

\section{Project Description}

The main scope of this work is to:

1. develop a novel tomographic ultrasonic technique to obtain the real-time distribution of acoustic velocity and flow velocity;

2. use nuclear magnetic resonance imaging (NMRI) to measure velocity profiles and rheological properties of complex fluids and suspensions;

3. establish a facility for making laser Doppler velocimetry (LDV) measurements that can be

The overall goal is to obtain real-time rheology and solids concentration within a solid-liquid suspension flowing in a pipeline. To nondestructively obtain the rheology of the fluid flowing in a pipe, accurate measurement of local shear rate distribution is required. This objective was met by using two real-time tomographic techniques: an ultrasonic Doppler velocimetry system and an NMRI system. The first method combines the existing state-of-the-art ultrasonic velocimetry technology base with a novel tomographic concept to non-intrusively obtain high resolution acoustic and flow velocity profile at a section of the flow field. The acoustic velocity profile provides a means of improving the flow velocity measurement accuracy. These data are also, used to yield the profile of solids concentration. In addition, the volumetric flow rate was determined from integration of the velocity profile. From the knowledge of the concentration profile the mass flow rate can also be determined, Similar work was undertaken for the NMNRI system. In this case, single phase Newtonian fluids have been used to model complex rheological behavior. Finally, a LDV system has been purchased and set - up in the laboratory at UC Davis. 


\section{Technical Accomplishments: Accoustic Doppler Velocimetry}

A fully developed flow in a circular pipe was generated using the appropriate hydraulic system. The velocity profile for this particular condition, if the flow is laminar and Newtonian, would be parabolic. As we have implemented this technique, the ultrasonic signal is transmitted into the pipe and return signal is received by the same transducer and supplied to t\&e data acquisition system. The processed signals are converted into a velocity profile. We have been able to accurately image steady laminar pipe flow (Reynolds number 400) of a solution of propylene glycol which is a Newtonian fluid. The velocity profile was parabolic, as expected.

Similar results were obtained for the flow of a Carbopol solution, which is a pseudoplastic fluid' with a flow behavior index of $n=0.47$. With such high level of shear-thinning behavior, the velocity profile showed a plug-like regime in the core. Indeed, it was observed that as the velocity was decreased the size of the plug increased in the radial direction, as has been reported by other investigators. Therefore, qualitatively, we believe the ultrasonic measurements are providing the correct results. Further tests were performed to investigate the uncertainty obtained in the measurements.

\section{Technical Accomplishments: N M R I}

We have shown that for a variety of single phase non-Newtonian fluids, it is possible to accurately determine the shear viscosity as a function of shear rate. These experiments are similar to those used for the acoustics work. We establish steady flow in a tube of circular cross section and determine the velocity profile. From a pressure drop measurements, we can find the local shear stress. The local shear rate is determined by differentiating the velocity profile. The viscosity at a point in the flow is found by dividing the shear stress by the shear rate. In principle, the range of shear rates in any experiment is zero at the tube center to a maximum at the tube wall.

The fluids that were used for these experiments included those that exhibited strong shear thinning behavior over the entire range of shear rates tested and also 'showed a range of shear rates where the fluid was Newtonian. NMRI is able to successfully delineate both regimes of behavior. Furthermore, we have found correlations that permit us to design NMRI experiments a priori given the desired range of shear rates and the mean flow conditions.

\section{Technical Accomplishments - Laser D oppler Velocimetry}

A closed-loop flow system has been designed and constructed to study fully developed pipe flow including laminar and turbulent regimes up to a Reynolds number of 20,000 with water as the test fluid. The test fluid is fed by gravity from an elevated tank through a valve and a rotameter into a specially constructed glass tube, oriented horizontally. The tube has an inside diameter of one inch with smooth interior walls over its entire length of fifteen feet. Fluid entering the tube passes trough a matrix of drinking straws to produce a uniform velocity profile. At the exit of 
the tube, the test fluid flows into a catch tank. A pump is used to drive a steady circulation of fluid between the catch and supply tanks. The pump, together with overflow channels near the top of the supply tank, provides for a constant level of fluid in the supply tank, and thus a constant supply pressure.

Velocities in the pipe will be measured at an axial. station about one foot from the exit of the, glass pipe, where the flow should be fully developed, using a laser Doppler velocimeter (LDV). The LDV system represents the state of the art, having the greatest resolution of analog-to-digital signal conversion Of available systems. Mean valves and turbulence intensities of the streamwise component of velocity will be obtained over a range of radial position across the pipe. The data will be used as a benchmark for comparison with measurements of the same quantities using nuclear magnetic resonance (NMR), techniques.

Construction of the flow system is nearly complete. The constant level tank system is in place and functioning, Fabrication of the glass tube is complete, and a sacrificial sample has shown that the interior wall is smooth to within 0.001 inch. At present, the main components of the flow system are being joined together, and the return loop is being constructed. The LDV system is in place and has been tested. Measurements will begin shortly. 